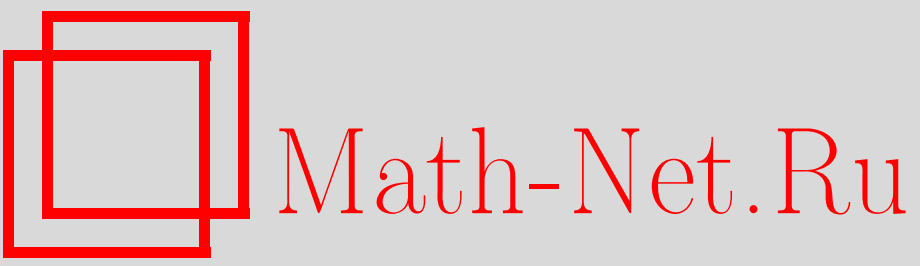

И. В. Полтерович, А. И. Шнирельман, Асимптотический подконус плоскости Лобачевского как пространство функций, УМН, 1997, том 52, выпуск 4, 209210

DOI: https://doi.org/10.4213/rm873

Использование Общероссийского математического портала Math-Net.Ru подразумевает, что вы прочитали и согласны с пользовательским соглашением http://www . mathnet.ru/rus/agreement

Параметры загрузки:

IP : 54.196 .121 .252

26 апреля 2023 г., $12: 19: 21$ 


\title{
АСИМПТОТИЧЕСКИЙ ПОДКОНУС ПЛОСКОСТИ ЛОБАЧЕВСКОГО КАК ПРОСТРАНСТВО ФУНКЦИЙ
}

\author{
И. ПОЛТЕРОВИЧ, А. ШНИРЕЛЬМАН
}

Пусть $X, X_{0}$ - метрические пространства с метриками $d_{X}, d_{X_{0}}$ соответственно. Нам потребуются следующие определения (см. [1]):

ОПРЕДЕЛЕНИЕ 1 . Пусть $x_{0}, x_{1} \in X_{0}$ и $a=d_{X_{0}}\left(x_{0}, x_{1}\right)$. Геодезическим сегментом в $X_{0}$, соединяющим точки $x_{0}$ и $x_{1}$, называется изометрическое вложение $g:[0, a] \rightarrow X_{0}$, такое что $g(0)=x_{0}, g(a)=x_{1}$. Пространство $X_{0}$ назьвается геодезическим, если любые две его точки $x_{0}, x_{1}$ можно соединить геодезическим сегментом (единственность не требуется).

ОПРЕДЕЛЕниЕ 2. Метрическое пространство $X_{0}$ назьвается вещественным деревом, если оно удовлетворяет следующим двум условиям:

1) для любых двух его различных точек существует единственный геодезический сегмент, соединяющий их;

2) если два геодезических сегмента $[a, b],[b, c]$ имеют ровно одну общую граничную точку $b$, то их объединение также есть геодезический сегмент.

М. Громовым было введено понятие асимптотического подконуса метрического пространства (см. [1], [2]):

ОПРЕДЕЛЕНИЕ 3 . Метрическое пространство $\left(X_{0}, d_{X_{0}}\right)$ назьвается асимптотическим поджонусом метрического пространства $\left(X, d_{X}\right)$, если оно является геодезическим и обладает следуюшим свойством: для любого конечного набора $\left(x_{1}, x_{2}, \ldots, x_{n}\right)$ из $n$ точек пространства $X_{0}$ существуют последователшность $\varepsilon_{i} \rightarrow 0$ и последовательность наборов $\left(x_{1}^{i}, x_{2}^{i}, \ldots, x_{n}^{i}\right)$ из $n$ точек пространства $X$, такие что

$$
\lim _{i \rightarrow \infty} \varepsilon_{i} \cdot d_{X}\left(x_{j}^{i}, x_{k}^{i}\right)=d_{X_{0}}\left(x_{j}, x_{k}\right), \quad j, k=1, \ldots, n .
$$

Понятие асимптотического подконуса является упрощением понятия асимптотического конуса метрического пространства (см. [3]). Каждое из них возникло в результате попыток охарактеризовать структуру метрического пространства на "бесконечности" (см. [1], [3]). Очевидно, любое подмножество асимптотического подконуса, являющееся геодезическим подпространством, само есть асимптотический подконус.

Если $X$ имеет конечный диаметр, то всякий его асимптотический подконус есть точка. Для неограниченных метрических пространств возникает вопрос об описании их асимптотических подконусов. Простейшим примером пространства, для которого этот вопрос интересен, служит плоскость Лобачевского. Она принадлежит к классу гиперболических пространств, для которых, как было доказано М. Громовым, любой асимптотический подконус есть вещественное дерево. Однако, дажке для плоскости Лобачевского про асимптотические подконусы практически ничего больше неизвестно.

Плоскость Лобачевского является однородньгм пространством, поэтому естественно искать асимптотические подконусы, обладающие тем же свойством. Всякий такой подконус есть вещественное дерево, ветвящееся в каждой своей точке. Главной целью работы является построение примера однородного асимптотического подконуса плоскости Лобачевского. Оказьвается, таким примером может служить метрическое пространство, чьи элементы суть вещественные непрерывные функции с ограниченньм носителем, расстояние между которыми вводится некоторым специальным образом:

ОПРЕДЕЛЕНИЕ 4 . Пусть $S$-пространство непрерьвных вещественных функций $f(t)$, каждая из которых задана на некотором конечном интервале $\left[0, \rho_{f}\right], 0 \leqslant \rho_{f}<\infty$, причем $f(0)=0$. Определим на $S$ метрику $d_{S}$ следующим образом:

$$
d_{S}\left(f_{1}, f_{2}\right)=\left(\rho_{f_{1}}-c\right)+\left(\rho_{f_{2}}-c\right)
$$

Работа второго автора вьполнена при поддержке французско-израильской программы научного и технического сотрудничества PICS № 296. 
где

$$
c=\sup \left\{t \mid f_{1}\left(t^{\prime}\right)=f_{2}\left(t^{\prime}\right) \forall t^{\prime}<t\right\} .
$$

Число $c$ назьвается моментом разделения между функциями $f_{1}, f_{2}$.

Расстояние (2) действительно превращает пространство $S$ в вещественное дерево, ветвящееся в каждой точке, причем мощность множества ветвей, исходящих из каждой точки (или, как еще говорят, число ветвления в каждой точке), есть континуум.

Следующая теорема является основным результатом работы:

ТЕОРема 1. Пространство S есть асимптотический подконус плоскости Лобачевского.

В доказательстве теоремы 1 (см. [4]) рассматривается модель Пуанкаре плоскости Лобачевского в круге, вводятся гиперболические полярные координаты. Оказьвается, что каждому конечному набору функций пространства $S$ можно сопоставить такой набор последовательностей точек плоскости Лобачевского, что для каждой последовательности радиальные координаты ее точек стремятся к бесконечности, а угловые экспоненциалњно сходятся к нулю. При этом попарные разности между скоростями сходимости к нулю угловых координат определяются моментами разделения соответствующих пар функций из $S$. Для осуществления перехода от плоскости Лобачевского к пространству $S$ строится вспомогательное метрическое пространство $D$. Оно состоит из всех вещественных функций $f(t)$, определенных на конечных полуинтервалах $\left[0, \rho_{f}\right)$, $0<\rho_{f}<\infty$, и таких, что $f(0)=0, f(t)=0$ всюду за исключением не более чем конечного числа точек. Метрика на $D$ определяется той же формулой, что и на $S$. Можно показать, что $D$ также есть асимптотический подконус плоскости Лобачевского.

Вернемся к изучению свойств пространства $S$. Имеет место важное утверждение:

Теорема 2. Асимптотический подконус $S$ есть однородное метрическое пространство.

Заметим, однако, что вопрос о связи между изометриями плоскости Лобачевского и пространства $S$ остается открытым.

Введем следующую характеристику вещественного дерева:

ОПРедЕЛЕниЕ 5. Вещественное дерево назьвается густым, если оно допускает изометрическое вложение любого вещественного дерева с не более чем счетным числом вершин.

Теорема 3. Пространство $S$ есть густое дерево.

В заключение приведем следствие теоремы 3 :

СлЕдСТвиЕ 1. Любое вещественное дерево с не более чем счетным числом вершин есть асимптотический подконус плоскости Лобачевского.

Доказательства приведенных утверждений содержатся в [4].

Авторы выражают глубокую благодарность М. Громову, Л. Полтеровичу и В. Бухштаберу за плодотворные обсуждения.

\section{СПИСОК ЛИТЕРАТУРЫ}

[1] Гис Э., де ля Арп П. (ред. ) Гиперболические группы по Михаилу Громову. М.: Мир, 1992. [2] Gromov M. Hyperbolic Groups // Essays in group theory / ed. S. M. Gersten. Berlin: Springer-Verlag, 1987. P. 75-263. [3] Gromov M. Asymptotic invariants of infinite groups // Geometric group theory. Vol. 2 (Sussex, 1991). London Math. Soc. Lecture Note Ser. 182. Cambridge: Cambridge Univ. Press, 1993. P. 1-295. [4] Polterovich I., Shnirelman A. Asymptotic subcones of the hyperbolic plane // Preprint: Tel Aviv University, 1997. P. 1-14.

Московский государственный университет им. М.В.Ломоносова, Институт Вейцмана (Израиль), 